\title{
AULA INVERTIDA COMO MÉTODO DE ENSEÑANZA EN LA UNIDAD DIDÁCTICA REACCIONES QUÍMICAS DE QUINTO GRADO DEL NIVEL SECUNDARIO DOMINICANO
}

\author{
Flipped Classroom as a Teaching Method in the Didactic Unit Chemical \\ Reactions of the Fifth Grade of the Dominican Secondary Level
}

Sergio Jato-Canales ${ }^{1}$

sjato@isa.edu.do

\section{Santo Fausto-Frías ${ }^{1}$}

santofausto3@gmail.com

Juan De Dios Domínguez-Liriano ${ }^{1}$ juandominguezliriano@gmail.com

${ }^{1}$ Universidad ISA. República Dominicana

Fecha de recepción: 10/10/2019

Fecha de aprobación: 1/06/2020

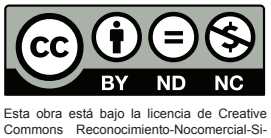

ISSN (impreso): 2636-2139

ISSN (en línea): 2636-2147

Sitio web: https://revistas.isfodosu.edu.do/recie

\section{Resumen}

El aula invertida es un enfoque que permite al estudiantado obtener información en un tiempo y lugar que no requiere la presencia física del profesor, cambiando de esta manera sus roles tradicionales. En el proceso de enseñanza de la química, los docentes se ven obligados a impartir contenidos que, por la falta de tiempo, no cumplen los parámetros curriculares establecidos, lo que afecta el desarrollo de los estudiantes y produce confusiones y mala interpretación. La presente investigación tiene como objetivo evaluar el impacto de la implementación del aula invertida como método de enseńanza para el desarrollo cognitivo en la unidad didáctica Reacciones Químicas de los discentes del nivel secundario dominicano. Este estudio se realiza bajo el enfoque cuantitativo, dirigido a estudiantes de quinto grado del nivel secundario, de los cuales se extrae una muestra de 58 alumnos divididos en dos grupos (grupo de control y grupo experimental), del Distrito Educativo 08-05, caracterizado por la aplicación del nuevo Diseño Curricular Dominicano y la implementación del programa República Digital. Las técnicas utilizadas son la observación y la encuesta, empleando como instrumentos la rúbrica, la lista de cotejo, la bitácora, la prueba diagnóstica y la post-prueba de evaluación. Los resultados muestran que los promedios obtenidos en la post-prueba por el grupo experimental en cada uno de los indicadores son mayores en comparación con los promedios presentados en el diagnóstico, además de superar también los resultados finales del grupo de control, lo que evidencia que el aula invertida tiene un efecto positivo en el desarrollo de las habilidades cognitivas de los discentes.

Palabras clave: aprendizaje, aula inverti$\mathrm{da}$, desarrollo cognitivo, método de enseñanza.

\section{Abstract}

The Flipped Classroom is an approach that allows students to obtain information in a time and place without physical presence of the teacher, thus changing their traditional roles. In the chemistry teaching process, teachers are forced to teach content, which due to lack of time, does not meet established curricular parameters, affecting student development, causing confusion and misinterpretation. The objective of this research is to evaluate the impact of the implementation of the Flipped Classroom, as a teaching method for cognitive development in the didactic unit Chemical Reactions of the Dominican secondary level students. This study is carried out under the quantitative approach, aimed at fifth-grade students at the secondary level, from which a sample of 58 pupils is extracted, divided into two groups (control group and experimental group), from the Educational District 08-05, characterized by the application of the new Dominican Curricular Design and the implementation of the Digital Republic program. The techniques used are observation and survey, using the rubric, checklist, log, diagnostic test and evaluation post-test as instruments. The results show that the averages obtained in the posttest by the experimental group in each of the indicators are higher compared to the averages presented in the diagnosis, in addition to also exceeding the final results of the control group, evidencing that the Inverted classroom it has a positive effect on the development of cognitive abilities of learners.

Keywords: learning, flipped classroom, cognitive development, teaching method. 


\section{Introducción}

En los últimos años, nuestra sociedad se ha visto caracterizada por los adelantos científicos y tecnológicos vinculados a todos los sectores organizacionales y, sobre todo, en el marco de la educación. La implementación de nuevos métodos de enseñanza ha generado profundas transformaciones y un estilo innovador en el que el docente deja de ser un transmisor de conocimientos ya acabados y toma conciencia de que su función es crear la posibilidad para que sus alumnos produzcan sus propios conocimientos dejando de ser pasivos y convirtiéndose en entes activos. La innovación educativa:

Es un acto deliberado y planificado de solución de problemas, que apunta a lograr mayor calidad en los aprendizajes de los estudiantes, superando el paradigma tradicional. Implica trascender el conocimiento academicista y pasar del aprendizaje pasivo del estudiante a una concepción donde el aprendizaje es interacción y se construye entre todos (UNESCO, 2016, p. 64).

No obstante, en la materia de Química, el profesorado se ve obligado a impartir contenidos, como en el caso de las reacciones químicas, que por su complejidad y la falta de tiempo no cumplen los parámetros curriculares establecidos, lo que afecta el aprendizaje y dominio de estos por parte del alumnado, con lo que se producen confusiones y mala interpretación de los mismos, favorecido, además, por la aplicación de modelos tradicionales, sin tomar en cuenta el ritmo de aprendizaje que posee cada estudiante. Según el Informe curricular de Pruebas Nacionales (Ministerio de Educación, 2017), los discentes del bloque Química General alcanzaron el promedio más alto de acierto (43.50\%) en las pruebas consolidadas, mientras que en Química Orgánica obtuvieron un $40.33 \%$. En ambos casos hubo un descenso de estos valores respecto al año 2016; en Química General habían alcanzado un promedio de acierto del 46.64 \% y en Química Orgánica del 42.32 \%. Es por ello que este estudio - cuyo objetivo principal consiste en evaluar el impacto de la implementación del aula invertida como método de enseñanza en la unidad didáctica Reacciones Químicas — pretende ser un soporte innovador a ser aplicado por docentes, para favorecer en los estudiantes un aprendizaje significativo, considerando a los mismos como seres capaces de regular lo que aprenden y a qué ritmo lo aprenden.

Con el fin de aprovechar al máximo el tiempo que dispone el profesorado a la hora de desarrollar y dar a conocer los contenidos de la unidad didáctica de las Reacciones Químicas, atendiendo al ritmo de aprendizaje de cada discente, se hace necesario la implementación de métodos como el aula invertida que, como su nombre indica, invierte los papeles de la metodología tradicional, en la que el docente se convierte en un guía para el estudiante en su proceso de aprendizaje en vez de ser un mero transmisor de los contenidos.

Partiendo de la problemática planteada, surge la siguiente interrogante: ¿Qué efecto tiene la implementación del aula invertida como método de enseñanza en el aprendizaje de los estudiantes de quinto grado del nivel secundario en la unidad didáctica Reacciones Químicas?

\section{Revisión de la literatura}

\subsection{Desarrollo cognitivo}

El desarrollo cognitivo es mucho más que la suma de nuevos hechos e ideas en un almacén existente de información: "Nuestros procesos mentales cambian de forma radical, aunque lenta, desde el nacimiento hasta la madurez, porque constantemente nos esforzamos por darle un sentido al mundo" (Piaget, 1970, citado por Woolfolk, 2010, p. 32). Es decir, suceden cambios ordenados graduales mediante los cuales los procesos mentales se vuelven más complejos.

En todo caso, al hablar de desarrollo cognitivo en esta investigación se están considerando y relacionando dos cosas: a) un conjunto de habilidades que tienen que ver, básicamente, con los procesos ligados a la adquisición, organización y retención, y b) el uso del conocimiento.

Es importante tener en cuenta esta doble referencia porque, siguiendo el claro planteamiento que 
hace Flavell (1985) en la introducción a su ya clásico libro sobre el desarrollo cognitivo, se pretende superar una visión más tradicional de la cognición que la restringía a los llamados procesos mentales superiores, relativos solo a los aspectos típicamente inteligentes y humanos (pensamiento, imaginación, creatividad, planificación, inferencia, solución de problemas, etc.).

Es por ello que dentro de esta investigación se citan las cuatros etapas o periodos de transición que atraviesa un sujeto durante su vida en su desarrollo cognitivo: "Piaget observó que los individuos pueden atravesar largos periodos de transición entre etapas, y que un individuo bien puede mostrar características de una etapa en una situación, pero características de otra etapa superior o inferior en otras situaciones" (Wooffolk, 2010, p. 33). A continuación, se mencionan las etapas establecidas por Piaget, asociadas a las edades de los sujetos: etapa sensoriomotriz, etapa preoperacional, etapa de operaciones concretas y etapa de operaciones formales, haciendo hincapié en la última al interés de los investigadores.

La etapa de operaciones formales comprende desde los 11 años en adelante. Las características básicas que presenta el sujeto son las siguientes: el reconocimiento de la estabilidad lógica del mundo físico; la noción de que los elementos pueden cambiar o transformarse y seguir conservando muchas de sus características originales; el entendimiento de que es posible revertir tales cambios. Así como también la capacidad para resolver problemas abstractos de forma lógica, habilidad directamente influida por la identidad, la comprensión y la reversibilidad, componentes básicos del razonamiento (Piaget, 1970).

\subsection{Evaluación del desarrollo cognitivo}

Hablar de la evaluación del desarrollo cognitivo no es sinónimo de calificar; es ir más allá, es referirse a la evaluación del aprendizaje alcanzado por los discentes, es decir, el dominio que presentan sobre los contenidos curriculares por medio del desarrollo de las habilidades cognitivas con la finalidad de ser practicados en situaciones de la vida real.
No obstante, la evaluación se concibe como una guía para los actores del proceso educativo que posibilita determinar la eficacia de la enseñanza y la calidad de los aprendizajes (Ministerio de Educación, 2016). En efecto, es un proceso continuo de investigación educativa que realiza el profesorado con la finalidad de identificar lo que los estudiantes han logrado y lo que les falta por lograr. Por esto cada unidad, proyecto o módulo que se inicia contempla los distintos tipos de evaluación: diagnóstica, formativa y sumativa.

\subsection{Aula invertida}

El desarrollo tecnológico alcanzado en la sociedad del nuevo siglo acompaña las tendencias educativas modernas. Al hablar del aula invertida como método de enseñanza para el desarrollo cognitivo nos referimos a un nuevo modelo pedagógico que contribuya a que los alumnos tengan un aprendizaje significativo, considerando a los mismos como seres capaces de regular lo que aprenden y a qué ritmo lo aprenden.

El aula invertida es:

un enfoque pedagógico en el que la instrucción directa se mueve desde un espacio de aprendizaje colectivo a un espacio de aprendizaje individual al estudiante, y el espacio de aprendizaje colectivo resultante, se transforma en un ambiente de aprendizaje dinámico e interactivo, donde el docente guía a los estudiantes a medida que él aplica los conceptos y participa creativamente en el tema (Quiroga, 2014, p. 1).

Los estudios realizados sobre el aula invertida demuestran en su mayoría un efecto significativo en las diferentes disciplinas, competencias, etc. Yarleque (2018) en la Universidad Cayetano Heredia de Lima (Perú), realizó una investigación titulada Flipped classroom y el efecto en las competencias transversales de los alumnos del curso de Electricidad y Electrónica Industrial, cuyo objetivo era analizar el efecto del modelo pedagógico Flipped classroom sobre las competencias transversales de los estudiantes del curso de Electricidad y Electrónica Industrial de la Facultad de Ingeniería Industrial y de Sistemas. Los resultados obtenidos en esta investigación revelaron que Flipped Classroom logró influir positivamente en las 
competencias transversales, resultados similares a los obtenidos por Eldy (2019) en su estudio Inverted classroom improves pre-university students understanding on basic topic of Physics: the preliminary study, en el que se muestra que hubo una diferencia significativa entre el grupo de control (aula tradicional) y el grupo experimental (aula invertida) en su puntaje anterior y posterior a las pruebas aplicadas en temas básicos de física preuniversitaria.

Cada vez más, docentes de diferentes áreas disciplinares están viendo el aula invertida como una metodología eficaz para favorecer el aprendizaje de contenidos más complejos. Por ejemplo, Joksimović et al. (2019), investigadores de la Facultad de Ingeniería Mecánica de la Universidad de Belgrado (Serbia), comprobaron en su investigación Implementation of Inverted Classroom Methodology in 3D Modeling Course cómo mejoraron las calificaciones de los estudiantes a la vez que aumentó su interés y colaboración durante el proceso de enseñanza aprendizaje.

Por otro lado, Sánchez-Cruzado (2017), de la Universidad de Málaga (España), realizó un estudio bajo el título Flipped classroom. La clase invertida, una realidad en la Facultad de Ciencias de la Educación de la Universidad de Málaga, cuyo objetivo fue analizar el grado de satisfacción del alumnado universitario en el uso de la metodología de aula invertida en distintas disciplinas. Los resultados obtenidos de la mencionada investigación radicaron en que el alumnado vio con satisfacción la introducción de una metodología que le proporcionaba mayor participación en el aula, que le facilitaba el acceso a contenidos didácticos en un formato más cercano, y que podía consultar cuando y como quisiera, adaptándose a sus necesidades y ritmos de aprendizaje.

Por su parte, Chaljub (2017), de la Pontificia Universidad Católica Madre y Maestra (República Dominicana), llevó a cabo un estudio titulado $E l$ b-Learning y la clase invertida para el desarrollo del aprendizaje activo, la autogestión y el pensamiento critico en el ámbito universitario, cuyo objetivo general fue el de desarrollar la autogestión de los aprendizajes y el pensamiento crítico a través de la clase invertida y trabajo colaborativo en estudiantes de la carrera de educación. Entre los hallazgos obtenidos se encontraron la valoración positiva de la clase invertida y la potenciación del desarrollo de los aprendizajes activos, tanto de forma individual como colectiva.

Bergmann y Sams (2012) realizaron un estudio con la finalidad de aplicar flipped classroom como solución para evitar que sus alumnos perdieran clases de química por alguna enfermedad, pérdida de tiempo al viajar desde lugares lejanos hasta la escuela y otros factores. Comprobaron que, con este nuevo modelo, los alumnos obtenían un mejor aprendizaje y por ende mejoraban sus calificaciones.

El aporte de los estudios citados como antecedentes a esta investigación reside en que la implementación de nuevos métodos de enseñanza puede impactar positivamente en la metodología del profesorado y en el aprendizaje de los alumnos, contribuyendo a que estos, como principales actores del proceso de enseñanza-aprendizaje, desempeñen el rol deseado en la actualidad: el docente como guía y facilitador, y el estudiante como un ente activo capaz de construir su propio aprendizaje.

Los recientes avances de la tecnología han permitido desarrollar potentes entornos de aprendizaje con tecnología mejorada, a pesar de que este nuevo modelo podría no basarse en la tecnología, es gracias a ella que se ha podido potenciar, y la misma es un componente clave en el modelo. Es evidente que la tecnología es un vehículo para el aprendizaje, pero es incuestionable que nos permitirá realizar el proceso de una manera más rápida y eficiente siempre y cuando se utilice de forma adecuada. Tal como expresa Cruz (2018) en su estudio Impacto del uso de las TIC en la educación superior dominicana, para formar excelentes profesionales competentes para la sociedad del conocimiento, las instituciones de educación dominicanas deben actualizar su plan curricular fundamentado en las TIC, adquirir y renovar su plataforma tecnológica, adiestrar y capacitar su personal docente e integrar las TIC como eje transversal a todos los programas para ser más eficaz y eficiente en el proceso enseñanza-aprendizaje. El modelo 
tradicional de educación presenta al maestro o educador impartiendo una clase en un aula, la cual sigue la secuencia "objetivos-introducción-desarrollo" con docentes explicando y alumnos escuchando. El limitado tiempo restante se dedica a dudas y a asignar tareas al alumnado, una actividad casi siempre individual en horarios fuera del tiempo de la clase (Muñoz, 2014).

El modelo de aula invertida implica que:

- el alumno recibe material (audio, video, etc.) que le permite estudiar los "objetivos-introducción-desarrollo" del tema antes de la clase y a su propio tiempo (en su casa). El material está enfocado al posterior trabajo en equipo;

- cuando es tiempo de la clase, todos los alumnos ya tienen los antecedentes, la clase ya no se centra en explicaciones teóricas, sino en actividades, ejercicios, prácticas; en resumen, poner en práctica mediante grupos el conocimiento.
Y se caracteriza por promover:

- mayor protagonismo del alumno en su propio aprendizaje, quien deja de ser un mero espectador de las explicaciones del profesor en clase. Es posible aplicar este método en edades del alumnado, áreas y niveles educativos distintos, lo cual hace que sea flexible;

- ahorro de tiempo y dinamismo para intercambiar opiniones y poner en práctica el aprendizaje a través de las actividades pautadas, gracias al mayor tiempo del que disponen los docentes para explicar, ayudar y evaluar los conceptos de mayor complejidad con la ayuda de la tecnología (base fundamental para el desarrollo de esta metodología).

A continuación, en la Tabla 1 se expone de un modo comparativo el rol asumido por docentes y estudiantes durante sesiones bajo métodos tradicionales y bajo el método de aula invertida.

Tabla 1. Rol del docente y del discente en métodos tradicionales y en aula invertida

\begin{tabular}{ll}
\hline \multicolumn{1}{c}{ Clase tradicional } & \multicolumn{1}{c}{ Clase invertida } \\
\hline Rol del docente en clase & \\
\hline Es el centro del proceso de enseñanza-aprendizaje, es decir, es un & Facilita material didáctico, asigna las actividades teóricas y prácticas, \\
sujeto activo conocedor del tema, un buen orador, sabe, informa e & asesora a los alumnos, responde preguntas de manera individual o \\
induce conocimientos acabados, resuelve ejemplos, es el único se- & en grupos pequeños, ofrece realimentación, vuelve a explicar con- \\
leccionador y organizador de contenidos, métodos, técnicas, mate- & ceptos a quienes lo requieran, es decir, dedica poco tiempo a guiar \\
riales, etc. & de forma individual al estudiante mientras aplica el conocimiento ad- \\
& quirido.
\end{tabular}

Rol del docente en casa

Prepara la clase centrada en sus objetivos, como único conocedor del tema, sin brindar oportunidades a los discentes para exponer sus ideas e intervenciones críticas.
Prepara la clase en función de los objetivos, incluye ejemplos, preguntas a través de audio, videos, artículos, etc.

Rol del alumnado en clase

Permanece sentado, toma apuntes, presta atención y realiza algunas preguntas, es decir, es un agente pasivo, con poca independencia cognoscitiva y pobre desarrollo del pensamiento teórico. Realiza muy pocas actividades de carácter práctico, pues la labor fundamental la ejerce el profesorado a través de la explicación.
Resuelve sus dudas, promueve debates, profundiza con aplicaciones prácticas, colabora entre pares y trabaja en grupo. En el aula es concebido como un agente activo, es capaz de construir sus propios conocimientos mediante la síntesis de información, la integración de competencias y la resolución de problemas. 


\section{Rol del alumnado en casa}

Repasa los apuntes de clase, realiza ejercicios de manera individual de acuerdo con el ritmo y el modo en que el docente realiza el proceso de enseñanza- aprendizaje.
Visualiza el material didáctico en general que ha sido establecido por el docente, lo procesa, toma apuntes, lo analiza, realiza un resumen, responde a las preguntas planteadas, etc.

Fuente: Adaptado a partir de Yarquele (2018).

\subsection{Aula invertida para la enseñanza-aprendizaje de la química}

La Química es la "ciencia que estudia la estructura, propiedades y transformaciones de los cuerpos a partir de su composición” (RAE, 2020). Debido a su condición de contemplar contenidos abstractos y sumada esta al necesario manejo de simbología y lenguaje científico, ha sido una asignatura considerada difícil de comprender. Intentar cumplir con todos los contenidos e indicadores de logro plasmados en sus planificaciones se torna en muchas ocasiones un reto para el profesorado de ciencias. De este modo surgen metodologías que intentan facilitar el aprendizaje de los discentes combinando recursos y medios que permitan una mayor comprensión de los contenidos. Por ejemplo, Bastidas (2020), en su investigación Aplicación del modelo pedagógico: aula invertida y su incidencia en el aprendizaje significativo de química en los estudiantes del primer año de B.G.U. de la U.E.M. "Sebastián de Benalcázar" de la ciudad de Quito, período 2018-2019, concluye que el aula invertida permite la construcción de los conocimientos, el intercambio de actividades y la realización de trabajos individuales o en grupo, fuera del aula de clase y dedicación de más tiempo a la realización de ejercicios prácticos, la resolución de dudas y problemas, debates, prácticas de laboratorio, evaluación, $\mathrm{y}$ actividades de reforzamiento de un determinado tema de química. Otro aspecto importante es el expuesto por Eichler y Peeples (2019) en su estudio Flipped Classroom Learning Environments in General Chemistry: What Is the Impact on Student Performance in Organic Chemistry? Estos autores afirman que quizá su hallazgo más importante es el grado de mejora estadísticamente significativo en el desempeño de los estudiantes menos preparados académicamente al implementar la metodología de aula invertida.
La utilización de las TIC en el aula de clase y fuera de ella constituye un elemento importante de la formación académica del estudiantado en materia de Química, tal como expresa Salazar (2019) en su investigación Aula invertida como metodología educativa para el aprendizaje de la quimica en educación media, en la cual concluyó que la articulación de las TIC con la metodología de aula invertida mejoró el trabajo colaborativo, la participación y la comunicación entre estudiantes y entre estudiantes y docente.

Por otro lado, la flexibilidad que permite esta metodología en cuanto a los enfoques que se pueden utilizar en la enseñanza la convierte en una herramienta poderosa para lograr resultados a corto plazo, ya que tiene una gran aceptación por parte de estudiantado, tal como indican Sierra et al. (2018) en su investigación titulada Evaluación del uso del método flipped classroom o aula invertida en el aprendizaje de la quimica: estudio de caso en la Institución Educativa Lacides C. Bersal de Lorica, en la que concluyeron que la asimilación de conceptos que no parecen relacionarse con el mundo cotidiano de los discentes se logró por su presentación adecuada con herramientas que simulan el fenómeno estudiado y que por medio de flipped classroom estos recursos podían estar a su disposición y ser retroalimentados por el docente, permitiendo un aprendizaje personalizado.

Sin embargo, no siempre es posible la implementación de este tipo de metodologías de un modo completamente exitoso. En un reciente estudio realizado por Broman y Johnels en 2019 titulado Flipping the class - University chemistry students' experiences from a new teaching and learning approach, se indica que existen problemas en cuanto a la aplicación de estas, ya que no alcanzan a despertar la motivación de los discentes por la asignatura y por ende su proceso de aprendizaje se convierte en algo mecánico. 


\section{JATO-CANALES • FAUSTO-FRÍAS • DOMÍNGUEZ-LIRIANO}

Aula invertida como método de enseñanza en la unidad didáctica Reacciones Químicas de quinto...

Igualmente, los resultados confirmaron que la mayor dificultad de aprendizaje de los estudiantes se debe a la falta de motivación y la apropiación del lenguaje de la química.

\section{Método}

\subsection{Enfoque y tipo de estudio}

En la presente investigación, los grupos en los que se prueba la variable se encontraban formados sin haber realizado ningún tipo de selección aleatoria o proceso de preselección. La investigación se enmarca bajo un enfoque cuantitativo, centrado en el análisis y comparación estadística de los resultados de acierto y de nivel de rendimiento alcanzado por los estudiantes de los dos grupos bajo estudio.

Con el fin de dar respuestas a los objetivos planteados y a la pregunta conductora de la investigación, se utilizan la observación estructurada y la encuesta como técnicas de investigación. Para mayor rigurosidad, se emplean además los siguientes instrumentos de recolección de datos: prueba diagnóstica para la evaluación del nivel de desarrollo cognitivo presente en los estudiantes (PPD), rúbrica de evaluación para el nivel de diseño del plan de acción (END), lista de cotejo para la evaluación del nivel de implementación del aula invertida (ENI), bitácora de evaluación para el alcance y nivel de implementación del aula invertida (BNI), y post-prueba para la evaluación del desarrollo cognitivo alcanzado por los estudiantes tras la implementación del aula invertida (PPE).

A continuación, en las Tablas 2 y 3 se detalla la operacionalización de variables seguida durante este estudio. 
Revista Caribeña de Investigación Educativa | 2021, 5(1), 19-39

\section{Tabla 2. Operacionalización de variables}

\begin{tabular}{|c|c|c|c|c|}
\hline Objetivo general & Objetivos específicos & Variable & Indicadores & Instrumentos \\
\hline \multirow[t]{4}{*}{$\begin{array}{c}\text { Evaluar el impacto de la } \\
\text { implementación del aula } \\
\text { invertida como método de } \\
\text { enseñanza para el desa- } \\
\text { rrollo cognitivo en la uni- } \\
\text { dad didáctica Reacciones } \\
\text { Químicas de los estudian- } \\
\text { tes de quinto grado del } \\
\text { nivel secundario. }\end{array}$} & $\begin{array}{c}\text { Diagnosticar el desarrollo } \\
\text { cognitivo que presentan } \\
\text { los estudiantes de quinto } \\
\text { grado del nivel secunda- } \\
\text { rio en la unidad didáctica } \\
\text { Reacciones Químicas } \\
\text { antes de la implementa- } \\
\text { ción. }\end{array}$ & Desarrollo cognitivo & $\begin{array}{c}\text {-Habilidad de resolución } \\
\text { de problemas } \\
\text {-Nivel de comprensión } \\
\text { simbólica } \\
\text {-Nivel de comprensión del } \\
\text { lenguaje científico }\end{array}$ & Prueba diagnóstica (PPD) \\
\hline & $\begin{array}{c}\text { Diseñar un plan de } \\
\text { unidad centrado en los } \\
\text { contenidos de la unidad } \\
\text { didáctica Reacciones } \\
\text { Químicas para el desarro- } \\
\text { llo de las habilidades cog- } \\
\text { nitivas de los estudiantes } \\
\text { de quinto grado del nivel } \\
\text { secundario. }\end{array}$ & $\begin{array}{l}\text { Nivel de diseño del plan } \\
\text { de unidad }\end{array}$ & $\begin{array}{c}\text {-Fines y objetivos } \\
\text {-Organización de los } \\
\text { contenidos } \\
\text {-Actividades seleccio- } \\
\text { nadas } \\
\text {-Recursos y medios se- } \\
\text { leccionados } \\
\text {-Distribución del tiempo }\end{array}$ & $\begin{array}{c}\text { Rúbrica de evaluación } \\
\text { para el nivel de diseño } \\
\text { del plan de unidad (END) }\end{array}$ \\
\hline & $\begin{array}{l}\text { Implementar el aula in- } \\
\text { vertida como método de } \\
\text { enseñanza en la unidad } \\
\text { didáctica Reacciones } \\
\text { Químicas para el desarro- } \\
\text { llo cognitivo de los estu- } \\
\text { diantes de quinto grado } \\
\text { del nivel secundario. }\end{array}$ & Nivel de implementación & $\begin{array}{l}\text {-Rol asumido por el do- } \\
\text { cente } \\
\text {-Cobertura de la im- } \\
\text { plementación del aula } \\
\text { invertida } \\
\text {-Acceso de los recursos } \\
\text { tecnológicos }\end{array}$ & $\begin{array}{c}\text { Lista de cotejo para la } \\
\text { evaluación del nivel de } \\
\text { implementación (ENI) } \\
\text { Bitácora para evaluar } \\
\text { el alcance y el nivel de } \\
\text { implementación del aula } \\
\text { invertida (BNI) }\end{array}$ \\
\hline & $\begin{array}{c}\text { Evaluar el desarrollo } \\
\text { cognitivo alcanzado por } \\
\text { los estudiantes de quinto } \\
\text { grado del nivel secun- } \\
\text { dario, después de la } \\
\text { implementación del aula } \\
\text { invertida como método de } \\
\text { enseñanza en la unidad } \\
\text { didáctica Reacciones } \\
\text { Químicas. }\end{array}$ & Desarrollo cognitivo & $\begin{array}{c}\text {-Habilidad de resolución } \\
\text { de problemas } \\
\text {-Nivel de comprensión } \\
\text { simbólica } \\
\text {-Nivel de comprensión del } \\
\text { lenguaje científico }\end{array}$ & $\begin{array}{l}\text { Post-prueba de evalua- } \\
\text { ción (PPE) }\end{array}$ \\
\hline
\end{tabular}

Nota. Los indicadores propuestos para la variable desarrollo cognitivo han sido adaptados y diseñados con base en Diseño Curricular Nivel Secundario (Ministerio de Educación, 2016). 
JATO-CANALES • FAUSTO-FRÍAS • DOMÍNGUEZ-LIRIANO

Aula invertida como método de enseñanza en la unidad didáctica Reacciones Químicas de quinto...

Tabla 3. Objetos a medir por cada indicador

\begin{tabular}{ccc}
\hline Indicadores & Objetos a medir \\
\hline IN1. Habilidad de resolución de problemas & $\begin{array}{c}\text { Capacidad con la que el estudiante identifica una reacción química. } \\
\text { Grado en que el estudiante reconoce los reactivos presentes en una } \\
\text { reacción química. }\end{array}$ \\
$\begin{array}{c}\text { Grado en que el estudiante reconoce los productos presentes en una } \\
\text { reacción química. }\end{array}$ \\
$\begin{array}{c}\text { Destreza que muestra el estudiante para identificar una ecuación química } \\
\text { balanceada. }\end{array}$ \\
Capacidad que muestra el estudiante para balancear una ecuación \\
química.
\end{tabular}

\subsection{Participantes}

La población correspondiente para el desarrollo de esta investigación está compuesta por los estudiantes de quinto grado del nivel secundario de la República Dominicana pertenecientes al Distrito Educativo 08-05 donde se aplica la nueva actualización del Diseño Curricular Dominicano, revisado y actualizado mediante la ordenanza 1-2017, y donde también se implementa el programa "República Digital" iniciado en el año 2017.

El programa República Digital busca esquematizar, implementar y promover estrategias de las Tecnologías de la Información y la Comunicación (TIC) en el sistema educativo dominicano, con el objetivo de brindar a cada estudiante y a cada profesor de las escuelas públicas a nivel nacional el acceso universal y gratuito a dispositivos electrónicos para reducir la brecha digital y del conocimiento (Ministerio de la Presidencia, 2020).

Con la nueva revisión y actualización curricular, la Educación Secundaria del sistema educativo dominicano comprende seis niveles divididos en dos ciclos de tres años cada uno, con una duración total de seis ańos, el cual comienza a los 11 y termina a los 17 años de edad. La asignatura de Química se desarrolla en el $5^{\text {to }}$ grado de dicho nivel secundario.

La muestra seleccionada corresponde a un centro educativo del nivel secundario que cumple con todas las características mencionadas: aplicación del nuevo diseño curricular, pertenecer al programa República Digital y contar sus discentes con los medios tecnológicos requeridos para las actividades a realizar. La cantidad total de estudiantes correspondiente a $5^{\text {to }}$ grado es de 80 en el referido centro, los cuales se encuentran distribuidos en tres secciones $\left(5^{\text {to }} \mathrm{A}=27\right.$, $5^{\text {to }} \mathrm{B}=22$ y $5^{\text {to }} \mathrm{C}=31$ ), de los cuales se extrae una muestra de 58 discentes pertenecientes a $5^{\text {to }} \mathrm{A}=27 \mathrm{y}$ $5^{\text {to }} \mathrm{C}=31$. De estos, los 27 estudiantes de $5^{\text {to }} \mathrm{A}$ representan el grupo experimental en el que se implementa el método de aula invertida y los 31 estudiantes de $5^{\text {to }} \mathrm{C}$ representan el grupo de control en el que no se implementa el estímulo del aula invertida como método de enseńanza. Los mismos son seleccionados por la proximidad de la población que ambos grupos tienen, con una diferencia de cuatro sujetos. 
JATO-CANALES • FAUSTO-FRÍAS • DOMÍNGUEZ-LIRIANO

Aula invertida como método de enseñanza en la unidad didáctica Reacciones Químicas de quinto...

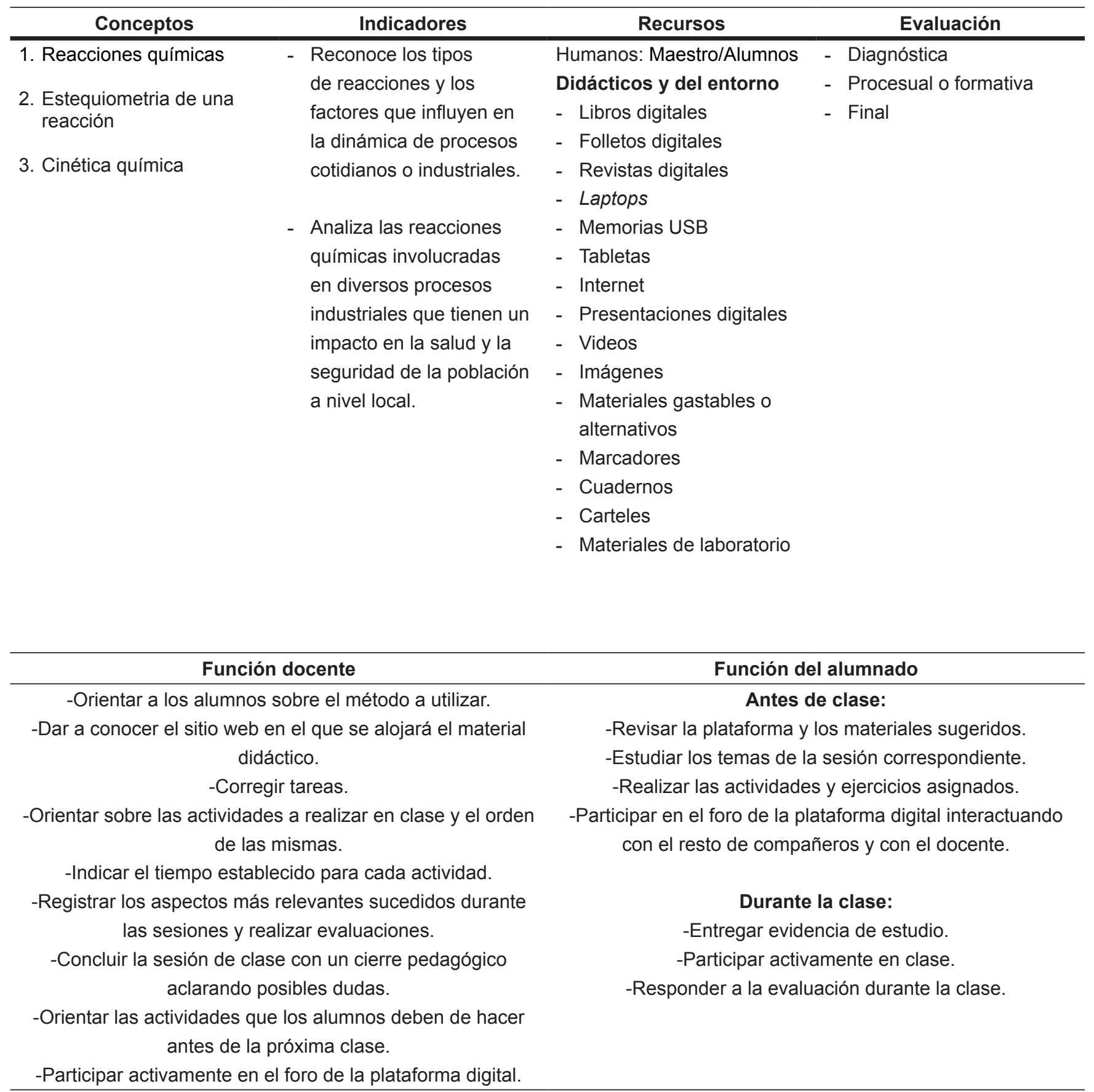


Revista Caribeña de Investigación Educativa | 2021, 5(1), 19-39

\begin{tabular}{|c|c|c|c|}
\hline \multicolumn{4}{|c|}{ Secuencia didáctica semana 1} \\
\hline Contenidos & Actividades & Indicadores de logro & Recursos \\
\hline $\begin{array}{l}\text { 1. Reacciones químicas } \\
\text { 1.1. Concepto básico de } \\
\text { reacciones químicas } \\
\text { 1.2. Estructura de las } \\
\text { reacciones químicas } \\
\text { 1.3. Clasificaciones de las } \\
\text { reacciones químicas } \\
\text { 1.3.1. Síntesis } \\
\text { 1.3.2. Descomposición } \\
\text { 1.3.3. Sustitución } \\
\text { 1.3.4. Doble sustitución } \\
\text { 1.4. Aplicaciones de las } \\
\text { reacciones químicas en } \\
\text { la vida cotidiana }\end{array}$ & 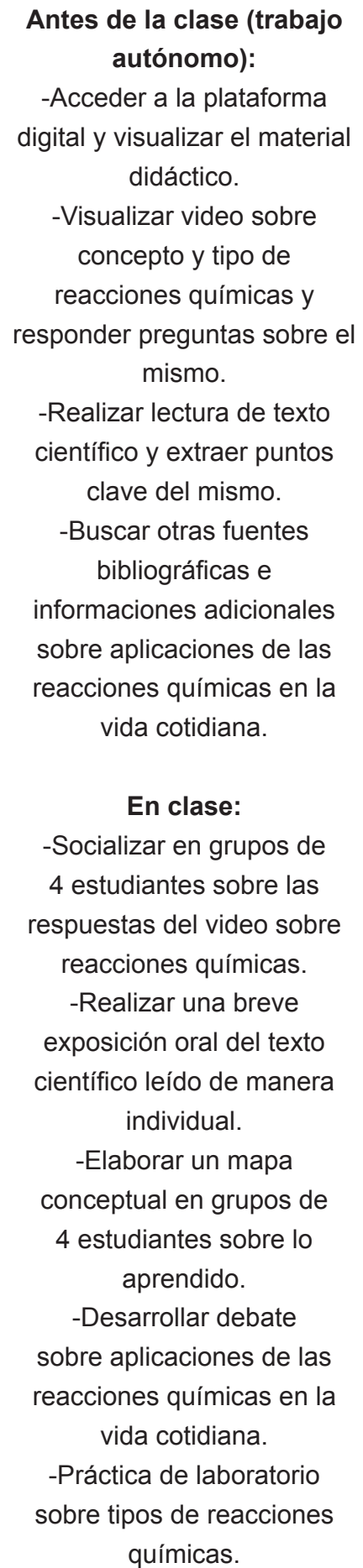 & $\begin{array}{l}\text {-Analiza el concepto de } \\
\text { reacciones químicas y } \\
\text { produce argumentos claros y } \\
\text { precisos. } \\
\text {-Reconoce los tipos de } \\
\text { reacciones y los factores que } \\
\text { influyen en la dinámica. } \\
\text {-Analiza las reacciones } \\
\text { químicas y su impacto en la } \\
\text { vida local de las personas. } \\
\text {-Clasifica los tipos de } \\
\text { reacciones químicas } \\
\text { de acuerdo con sus } \\
\text { características. } \\
\text {-Analiza las diferencias } \\
\text { existentes en cada una de } \\
\text { las reacciones químicas. } \\
\text {-Reconoce las características } \\
\text { que poseen en común las } \\
\text { reacciones estudiadas. }\end{array}$ & $\begin{array}{l}\text {-Plataforma digital Google } \\
\text { Classroom } \\
\text {-Vídeo sobre el concepto y } \\
\text { estructura de las reacciones } \\
\text { químicas } \\
\text {-Texto científico sobre } \\
\text { concepto y clasificación de } \\
\text { las reacciones químicas } \\
\text {-Libro de texto digital } \\
\text {-Material básico de } \\
\text { laboratorio }\end{array}$ \\
\hline
\end{tabular}


JATO-CANALES • FAUSTO-FRÍAS • DOMÍNGUEZ-LIRIANO

Aula invertida como método de enseñanza en la unidad didáctica Reacciones Químicas de quinto...

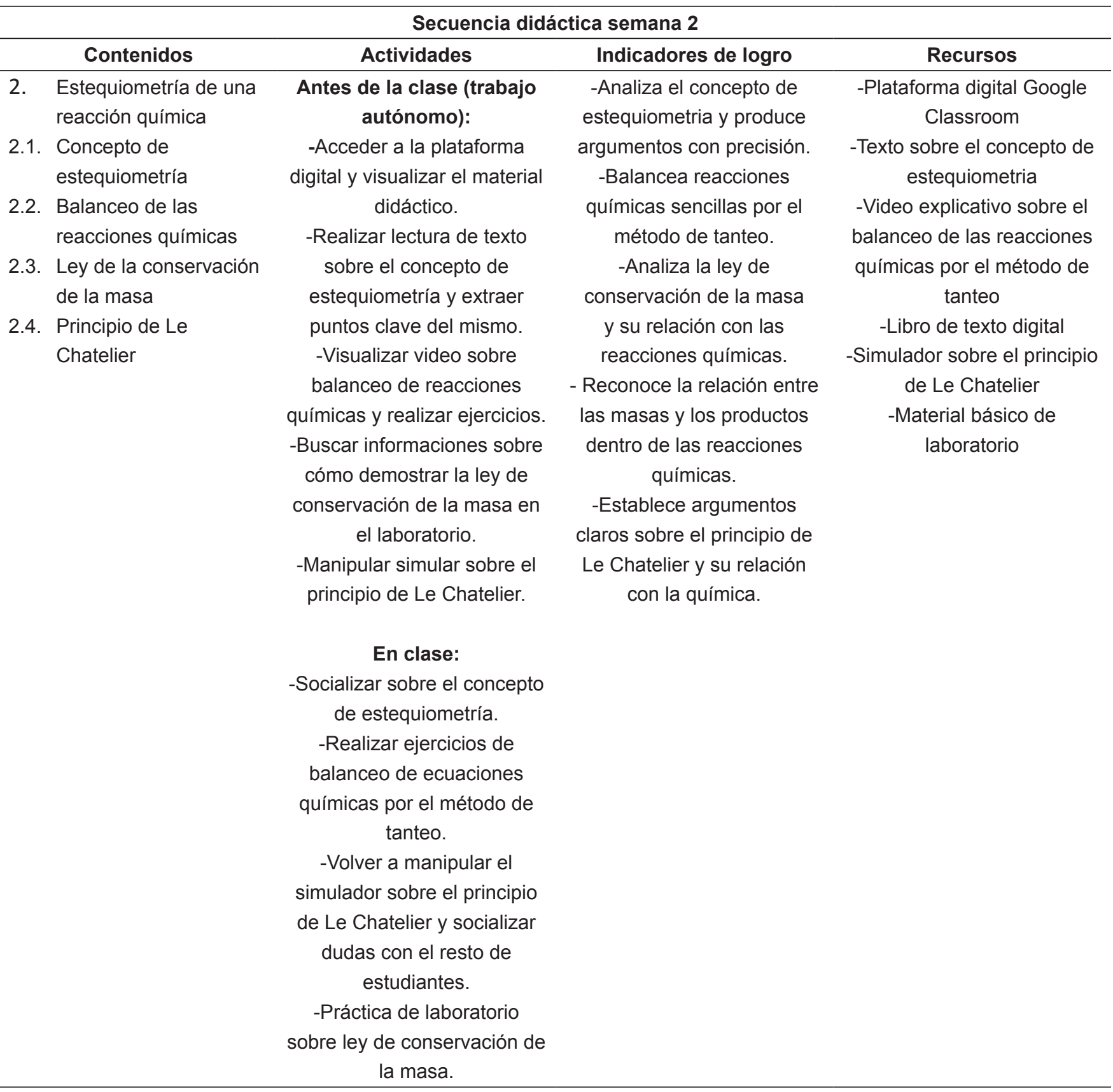


Revista Caribeña de Investigación Educativa | 2021, 5(1), 19-39

\begin{tabular}{|c|c|c|c|}
\hline \multicolumn{4}{|c|}{ Secuencia didáctica semana 3} \\
\hline Contenidos & Actividades & Indicadores de logro & Recursos \\
\hline $\begin{array}{l}\text { 3. Cinética química } \\
\text { 3.1. Concepto de cinética } \\
\text { química } \\
\text { 3.2. Factores que afectan la } \\
\text { cinética de una reacción } \\
\text { 3.3. Energía de activación } \\
\text { 3.4. Presencia de } \\
\text { catalizadores en una } \\
\text { reacción }\end{array}$ & 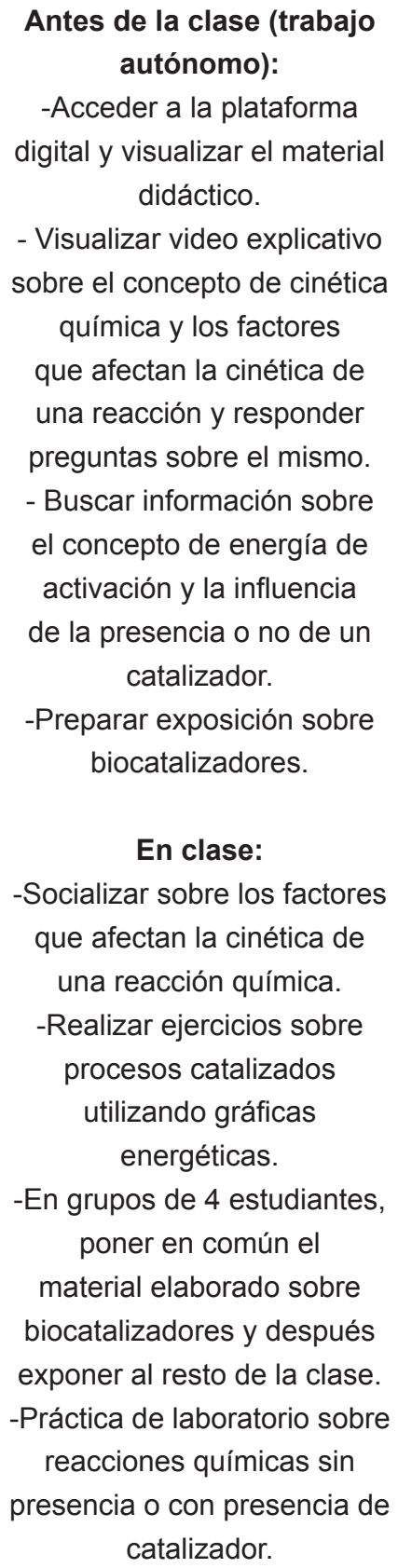 & $\begin{array}{l}\text {-Contrasta el concepto de } \\
\text { cinética química destacando } \\
\text { las características que } \\
\text { identifican a cada tipo de } \\
\text { reacción química, y produce } \\
\text { argumentos claros y } \\
\text { precisos. } \\
\text {-Reconoce los diferentes } \\
\text { factores que influyen en la } \\
\text { cinética de las reacciones } \\
\text { químicas. } \\
\text {-Analiza la energía de } \\
\text { activación de una reacción } \\
\text { química de acuerdo con la } \\
\text { cantidad de moléculas y } \\
\text { átomos presentes. } \\
\text {-Presenta argumentos sobre } \\
\text { los catalizadores y su efecto } \\
\text { en las reaccione químicas. }\end{array}$ & $\begin{array}{l}\text {-Plataforma digital Google } \\
\text { Classroom } \\
\text {-Video explicativo sobre el } \\
\text { concepto de cinética química } \\
\text { y los factores que afectan la } \\
\text { cinética de una reacción } \\
\text {-Gráficas energéticas de } \\
\text { procesos químicos con y sin } \\
\text { presencia de catalizadores } \\
\text {-Libro de texto digital } \\
\text {-Material básico de } \\
\text { laboratorio }\end{array}$ \\
\hline
\end{tabular}


- Ya diseńado y validado por expertos en química y pedagogía el plan de unidad, se procede a desarrollarlo a través de la implementación del aula invertida en el grupo experimental mediante la utilización de la plataforma virtual Google Classroom, teniendo los estudiantes acceso al material didáctico y la puesta en constancia de las actividades a realizar fuera y dentro del aula de clase. Por otro lado, para el grupo de control se desarrolla un segundo plan de unidad ajeno a la implementación del aula invertida, con base en los mismos contenidos, pero con diferentes actividades en las que se utilizan medios y recursos tecnológicos.

- Durante el desarrollo de las sesiones de clase se aplica la técnica de observación registrando en instrumentos como la lista de cotejo (ENI) y bitácora (BNI) los datos más relevantes sobre hechos y acciones observadas concernientes a cada uno de los indicadores relacionados al nivel de implementación del aula invertida.

- Agotados los contenidos con sus respectivas actividades de los planes de unidad, se procede a aplicar la post-prueba de evaluación (PPE) a ambos grupos bajo estudio (grupo de control y experimental) para constatar el desarrollo cognitivo alcanzado por estos.

\section{Resultados}

Para el análisis de los datos de esta investigación se procede de acuerdo con las técnicas e instrumentos utilizados: evaluación de la prueba diagnóstica (PPD) sobre el desarrollo cognitivo que presenta el estudiantado de quinto grado del nivel secundario en la unidad didáctica Reacciones Químicas, aplicada a los grupos experimental y de control, por indicadores de las variables, los cuales permiten medir la habilidad de resolución de problemas, el nivel de comprensión simbólica y el nivel de comprensión del lenguaje científico, nivelados sobre un máximo de un $100 \%$ de logro.
Del mismo modo, se analizan los datos de la post-prueba de evaluación (PPE) sobre el desarrollo cognitivo alcanzado por los discentes de quinto grado del nivel secundario, después de la implementación del aula invertida (grupo experimental), y grupo de control sin la aplicación del aula invertida como método de enseńanza en la unidad didáctica Reacciones Químicas.

Los resultados obtenidos de la prueba diagnóstica (PPD) y la post-prueba de evaluación (PPE) de $5^{\text {to }}$ A (grupo experimental) compuesto por 27 discentes y $5^{\text {to }} \mathrm{C}$ (grupo de control) compuesto por 31 discentes se tabulan utilizando Microsoft Office Excel, en el cual se crean varias tablas, en relación con ambos grupos y según el porcentaje de acierto y el nivel de logro alcanzado por indicador para cada discente. Del mismo modo, se promedia el número de acierto global de los estudiantes para aplicar posteriormente la estadística descriptiva, permitiendo la toma de decisiones acerca de la relación existente entre los datos recabados procedentes de cada grupo con la pregunta de investigación, y qué tan generalizables son estos resultados a un número mayor de sujetos de los que los que participaron en el estudio. Además, se realizan cruces dentro de los resultados obtenidos provenientes del grupo experimental y el grupo de control para una mayor interpretación y confiabilidad de los datos, con el fin de responder a los objetivos de la investigación.

A continuación, en la Tabla 5 se muestran los resultados obtenidos en el diagnóstico del desarrollo cognitivo que presentan los estudiantes de quinto grado del nivel secundario en la unidad didáctica Reacciones Químicas antes de la implementación. 
Revista Caribeña de Investigación Educativa | 2021, 5(1), 19-39

Tabla 5. Estadística descriptiva de los resultados obtenidos por los dos grupos en la prueba diagnóstica (PPD)

\begin{tabular}{|c|c|c|c|c|}
\hline \multirow[t]{2}{*}{ Indicador } & \multicolumn{2}{|c|}{ Grupo de control } & \multicolumn{2}{|c|}{ Grupo experimental } \\
\hline & Media & $\begin{array}{l}\text { Desviación } \\
\text { estándar }\end{array}$ & Media & $\begin{array}{c}\text { Desviación } \\
\text { estándar }\end{array}$ \\
\hline IN1 Habilidad de resolución de problemas & 46.58 & 21.02 & 40.59 & 23.54 \\
\hline IN2 Nivel de comprensión simbólica & 48.39 & 21.77 & 51.11 & 15.02 \\
\hline $\begin{array}{l}\text { IN3 Nivel de comprensión del lenguaje } \\
\text { científico }\end{array}$ & 53.13 & 21.81 & 54.04 & 19.38 \\
\hline Acierto global & 49.68 & 14.45 & 51.04 & 13.63 \\
\hline
\end{tabular}

En sentido general, el grupo experimental obtiene un $51.04 \%$ como promedio de acierto global, un valor superior al $49.68 \%$ de acierto global que alcanza el grupo de control. Sin embargo, ninguno de los grupos bajo estudio obtiene un puntaje mayor que el $70 \%$ (mínimo exigible) por cada indicador. En vista de los resultados, se puede determinar que el nivel de desarrollo cognitivo es muy parecido en ambos grupos, evidenciándose un equilibrio considerable en las destrezas demostradas en cada uno de los indicadores.

Del mismo modo, se registran y analizan los resultados de la evaluación del desarrollo cognitivo que alcanzan los estudiantes de quinto grado del nivel secundario, después de la implementación del aula invertida como método de enseńanza en la unidad didáctica Reacciones Químicas. A continuación, se detallan dichos resultados.

Tabla 6. Estadística descriptiva de los resultados de los dos grupos en la post-prueba de evaluación (PPE)

\begin{tabular}{ccccc}
\hline Indicador & \multicolumn{2}{c}{ Grupo de control } & \multicolumn{2}{c}{ Grupo experimental } \\
\cline { 2 - 5 } & Media & $\begin{array}{c}\text { Desviación } \\
\text { estándar }\end{array}$ & Media & $\begin{array}{c}\text { Desviación } \\
\text { estándar }\end{array}$ \\
\hline $\begin{array}{c}\text { IN1 Habilidad de resolución de } \\
\text { problemas }\end{array}$ & 61.03 & 22.86 & 75.29 & 20.92 \\
\hline IN2 Nivel de comprensión simbólica & 77.74 & 23.62 & 75.56 & 24.39 \\
\hline $\begin{array}{c}\text { IN3 Nivel de comprensión del lenguaje } \\
\text { científico }\end{array}$ & 46.61 & 17.82 & 66.74 & 19.93 \\
\hline Acierto global & 61.42 & 10.91 & 72.67 & 14.92 \\
\hline
\end{tabular}


En sentido general, el grupo experimental obtiene un $72.67 \%$ como promedio de acierto global, superando el $70 \%$ (mínimo exigible) y superando también el promedio del $61.42 \%$ de acierto global que alcanza el grupo de control (Tabla 6). Se evidencia una diferencia en el nivel de aprendizaje en ambos grupos, con avances de un estado menos equilibrado a un estado superior de equilibrio en el caso del grupo experimental en los indicadores IN1 e IN3, sin embargo, el grupo de control presenta dicho progreso en el indicador IN2.

Para una mayor claridad e interpretación, en la Tabla 7 se muestra la comparación de los resultados de cada uno de los grupos bajo estudio en la prueba diagnóstica (PPD) y en la post-prueba de evaluación (PPE).

Tabla 7. Comparación de resultados de cada uno de los grupos bajo estudio en la prueba diagnóstica (PPD) y post-prueba de evaluación (PPE)

\begin{tabular}{ccccc}
\hline \multirow{2}{*}{ Indicador } & \multicolumn{2}{c}{ Grupo de control } & \multicolumn{2}{c}{ Grupo experimental } \\
\cline { 2 - 5 } & PPD & PPE & PPD & PPE \\
\hline IN1 Habilidad de resolución de problemas & 46.58 & 61.03 & 40.59 & 75.29 \\
\hline IN2 Nivel de comprensión simbólica & 48.39 & 77.74 & 51.11 & 75.56 \\
\hline $\begin{array}{c}\text { IN3 Nivel de comprensión del lenguaje } \\
\text { científico }\end{array}$ & 53.13 & 46.61 & 54.04 & 66.74 \\
\hline Acierto global & 49.68 & 61.42 & 51.04 & 72.67 \\
\hline
\end{tabular}

Dado que el objetivo general de la investigación consiste en evaluar el impacto de la implementación del aula invertida como método de enseńanza para el desarrollo cognitivo en la unidad didáctica Reacciones Químicas de los estudiantes de quinto grado del nivel secundario, se procede a categorizar los resultados por niveles de rendimiento, cuatro en total, tomando en cuenta el promedio de cada uno de los indicadores que alcanzan los discentes que recibieron el estímulo del aula invertida como método de enseńanza (Tabla 8). Dichos niveles, en concordancia con el sistema de calificaciones escolares, están distribuidos y comprendidos de la siguiente manera:
- Nivel 0 para aquellos discentes que alcanzan un promedio de acierto de 0 a 69.99 (calificación de D).

- Nivel 1 para los discentes que alcanzan un promedio de acierto de 70 a 79.99 (calificación de C).

- Nivel 2 para aquellos discentes que obtienen un promedio de acierto de 80 a 89.99 (calificación de B).

- Nivel 3 para los discentes que alcanzan un promedio de acierto de 90 a 100 (calificación de A).

Tabla 8. Distribución de estudiantes del grupo experimental por niveles de rendimiento en la prueba diagnóstica (PPD) y en la post-prueba de evaluación (PPE)

\begin{tabular}{|c|c|c|c|c|c|c|c|c|c|c|c|c|}
\hline \multirow{3}{*}{$\begin{array}{c}\text { Indicador } \\
\text { Instrumento } \\
\begin{array}{c}\text { Nivel de } \\
\text { rendimiento }\end{array}\end{array}$} & \multicolumn{4}{|c|}{$\begin{array}{l}\text { IN1 Habilidad de resolución de } \\
\text { problemas }\end{array}$} & \multicolumn{4}{|c|}{ IN2 Nivel de comprensión simbólica } & \multicolumn{4}{|c|}{$\begin{array}{l}\text { IN3 Nivel de comprensión del } \\
\text { lenguaje científico }\end{array}$} \\
\hline & \multicolumn{2}{|c|}{ PPD } & \multicolumn{2}{|c|}{ PPE } & \multicolumn{2}{|c|}{ PPD } & \multicolumn{2}{|c|}{ PPE } & \multicolumn{2}{|c|}{ PPD } & \multicolumn{2}{|c|}{ PPE } \\
\hline & $\mathrm{n}$ & $\%$ & $\mathrm{n}$ & $\%$ & $\mathrm{n}$ & $\%$ & $\mathrm{n}$ & $\%$ & $\mathrm{n}$ & $\%$ & $\mathrm{n}$ & $\%$ \\
\hline Nivel 0 & 25 & 92.5 & 13 & 48.1 & 24 & 92.5 & 8 & 33.3 & 24 & 88.8 & 14 & 51.8 \\
\hline Nivel 1 & 0 & 0 & 0 & 0 & 0 & 0 & 1 & 0 & 1 & 3.7 & 7 & 25.9 \\
\hline Nivel 2 & 2 & 7.4 & 7 & 25.9 & 3 & 7.4 & 9 & 33.3 & 2 & 7.4 & 6 & 22.2 \\
\hline Nivel 3 & 0 & 0 & 7 & 25.9 & 0 & 0 & 9 & 33.3 & 0 & 0 & 0 & 0 \\
\hline
\end{tabular}




\section{Habilidad de resolución de problemas}

Se evidencia que el $92.5 \%$ de los estudiantes alcanza un promedio de acierto comprendido entre 0 y 69 en la prueba diagnóstica, presentando un nivel cero, mientras que en la post-prueba ese porcentaje disminuye hasta el $48.1 \%$. También es destacable el hecho de que al inicio de este estudio solamente el $7.4 \%$ de los estudiantes presenta niveles de rendimiento comprendidos entre el 2 y el 3, aumentando significativamente hasta el $51.8 \%$ al final de la implementación.

\section{Nivel de comprensión simbólica}

En este indicador sucede algo similar al anterior. Antes de la implementación del método, el 92.5\% de los estudiantes presenta un nivel 0 de rendimiento, disminuyendo prácticamente una tercera parte
(33.3 \%) ese valor al finalizar el proceso. Esta tendencia positiva se refleja también en el hecho de que el $66.6 \%$ logra alcanzar niveles 2 y 3 en la post-prueba de evaluación.

\section{Nivel de comprensión del lenguaje científico}

Este es el indicador en el que menos impacto positivo se logra, ya que ningún estudiante se posicionó en un nivel de rendimiento 3 , ni antes de la implementación ni después de la misma. Aun así, el $48.1 \%$ de los discentes alcanzan niveles de rendimiento 1 y 2 al culminar el proceso frente al $11.1 \%$ del inicio.

La Tabla 9 muestra la evaluación del nivel de implementación del aula invertida por parte de los docentes, dividida en secuencias didácticas según los indicadores y criterios a evaluar.

\section{Tabla 9. Evaluación del alcance y el nivel de implementación del aula invertida}

\begin{tabular}{|c|c|c|c|c|}
\hline \multirow[t]{2}{*}{ Indicadores } & \multirow[t]{2}{*}{ Criterios } & \multicolumn{3}{|c|}{ Observaciones } \\
\hline & & Secuencia 1 & Secuencia 2 & Secuencia 3 \\
\hline \multirow[t]{2}{*}{$\begin{array}{l}\text { 1. Cobertura de la } \\
\text { implementación del } \\
\text { aula invertida }\end{array}$} & $\begin{array}{l}\text { 1.1. El aula invertida } \\
\text { incluye a toda la pobla- } \\
\text { ción estudiantil del aula. }\end{array}$ & $\begin{array}{l}\text { En principio no incluye } \\
\text { toda la población estu- } \\
\text { diantil del aula, porque } \\
\text { no todos forman parte } \\
\text { de la plataforma digital. }\end{array}$ & $\begin{array}{l}\text { Durante el desarrollo } \\
\text { de esta unidad, sobre } \\
\text { todo en la secuencia } \\
\text { didáctica de estequio- } \\
\text { metría se evidencia que } \\
\text { los estudiantes poseen } \\
\text { al } 100 \% \text { el acceso y } \\
\text { participación al nuevo } \\
\text { método y actividades a } \\
\text { realizar. }\end{array}$ & $\begin{array}{l}\text { El acceso y participa- } \\
\text { ción de los estudiantes } \\
\text { se mantiene hasta al } \\
\text { final en un } 100 \% \text { de los } \\
\text { casos. }\end{array}$ \\
\hline & $\begin{array}{l}\text { 1.2. El aula invertida es } \\
\text { viable para contemplar } \\
\text { los componentes teóri- } \\
\text { cos y prácticos. }\end{array}$ & $\begin{array}{l}\text { De entrada, no es via- } \\
\text { ble para contemplar los } \\
\text { componentes teóricos } \\
\text { y prácticos, porque } \\
\text { los discentes no están } \\
\text { acostumbrados a tra- } \\
\text { bajar con el método de } \\
\text { aula invertida. }\end{array}$ & $\begin{array}{l}\text { Tomando en cuenta el } \\
\text { contenido a desarrollar, } \\
\text { el aula invertida pre- } \\
\text { senta viabilidad para } \\
\text { este contenido de este- } \\
\text { quiometría como para } \\
\text { otros más, y permite } \\
\text { contemplar claramente } \\
\text { los contenidos teóricos } \\
\text { y prácticos durante } \\
\text { todo el desarrollo del } \\
\text { proceso. }\end{array}$ & $\begin{array}{c}\text { Lo es, porque hay } \\
\text { muchas herramientas } \\
\text { que complementan } \\
\text { las diferentes páginas } \\
\text { web siendo viable para } \\
\text { el estudio de los com- } \\
\text { ponentes teóricos y } \\
\text { prácticos. }\end{array}$ \\
\hline
\end{tabular}


JATO-CANALES • FAUSTO-FRÍAS • DOMÍNGUEZ-LIRIANO

Aula invertida como método de enseñanza en la unidad didáctica Reacciones Químicas de quinto...

\begin{tabular}{|c|c|c|c|c|}
\hline \multirow[t]{2}{*}{ Indicadores } & \multirow[t]{2}{*}{ Criterios } & \multicolumn{3}{|c|}{ Observaciones } \\
\hline & & Secuencia 1 & Secuencia 2 & Secuencia 3 \\
\hline \multirow[t]{3}{*}{$\begin{array}{l}\text { 2. Acceso a los re- } \\
\text { cursos tecnológicos }\end{array}$} & $\begin{array}{l}\text { 2.1. Se especifica el } \\
\text { número de actividades } \\
\text { teóricas y prácticas que } \\
\text { requieren del uso de los } \\
\text { recursos tecnológicos } \\
\text { desarrolladas por los } \\
\text { estudiantes dentro de } \\
\text { su programación. }\end{array}$ & $\begin{array}{l}\text { Estos solo se especifi- } \\
\text { can en correspondencia } \\
\text { con las actividades a } \\
\text { realizar dependiendo } \\
\text { del contenido a ser } \\
\text { estudiado. }\end{array}$ & $\begin{array}{l}\text { Dentro de la plataforma } \\
\text { utilizada siempre se } \\
\text { especifica, incluyendo } \\
\text { el plan de unidad el } \\
\text { número de actividades } \\
\text { teóricas y prácticas que } \\
\text { requieren el uso de los } \\
\text { recursos tecnológicos } \\
\text { que realizan los es- } \\
\text { tudiantes, como es el } \\
\text { caso de simuladores y } \\
\text { más. }\end{array}$ & $\begin{array}{c}\text { Durante el desarrollo de } \\
\text { cada sesión presencial } \\
\text { y a distancia siempre } \\
\text { se especifican las ac- } \\
\text { tividades y recursos } \\
\text { tecnológicos a ser utili- } \\
\text { zados. }\end{array}$ \\
\hline & $\begin{array}{l}\text { 2.2. Se evidencia por } \\
\text { parte de los estudiantes } \\
\text { la utilización de softwa- } \\
\text { res y páginas web para } \\
\text { la realización de las } \\
\text { actividades asignadas } \\
\text { sobre el contenido de } \\
\text { reacciones químicas. }\end{array}$ & $\begin{array}{l}\text { No en todos los mo- } \\
\text { mentos, de igual forma } \\
\text { hay que tomar en cuen- } \\
\text { ta el nivel de motivación } \\
\text { e interés por parte de } \\
\text { los estudiantes y en su } \\
\text { relación con los conte- } \\
\text { nidos a trabajar. }\end{array}$ & $\begin{array}{c}\text { Sí se evidencia al } \\
100 \% \text { la utilización de } \\
\text { software y páginas web } \\
\text { para la realización de } \\
\text { las actividades asigna- } \\
\text { das. }\end{array}$ & $\begin{array}{l}\text { Siempre se evidencia la } \\
\text { utilización de diferentes } \\
\text { páginas web para la } \\
\text { realización de las activi- } \\
\text { dades asignadas. }\end{array}$ \\
\hline & $\begin{array}{c}\text { 2.3. Se evidencia una } \\
\text { frecuencia en la utiliza- } \\
\text { ción de los softwares } \\
\text { y páginas web en las } \\
\text { actividades a realizar } \\
\text { por los estudiantes en } \\
\text { clase. }\end{array}$ & $\begin{array}{l}\text { No con mucha frecuen- } \\
\text { cia si estas páginas no } \\
\text { son recomendadas por } \\
\text { el docente. }\end{array}$ & $\begin{array}{c}\text { Si se presenta una } \\
\text { frecuencia constante de } \\
\text { uso de software y pági- } \\
\text { nas web en las activida- } \\
\text { des a realizar por parte } \\
\text { de los estudiantes; } \\
\text { páginas y programas } \\
\text { confiables. }\end{array}$ & $\begin{array}{l}\text { Es muy evidente, sobre } \\
\text { todo cuando están bien } \\
\text { orientados y motivados. }\end{array}$ \\
\hline
\end{tabular}

\section{Discusión y conclusiones}

El estudio realizado refleja que la implementación del aula invertida impacta positivamente el desarrollo de las habilidades cognitivas de los discentes en el área de química, tal y como se evidencia en los resultados del grupo experimental en cada uno de los indicadores medidos (habilidades cognitivas de resolución de problemas, comprensión de la simbología y comprensión del lenguaje científico), en los que se denota un incremento significativo de los promedios de acierto y niveles de rendimiento de los alumnos y en los que la mayoría de estos obtienen un promedio superior al exigible para ese nivel de enseñanza con una variación mínima por indicador. Estos resultados corroboran los hallazgos de autores como Bastidas (2020), y refuerzan esta metodología como adecuada para la enseńanza-aprendizaje de determinados temas de química.

Considerando estos resultados después de la implementación del aula invertida como método de enseñanza, se concluye que cuando los estudiantes aprenden a través de nuevos métodos, junto con la retroalimentación continua y el cambio de roles, se promueve que aumenten el desarrollo de sus habilidades cognitivas y su motivación, inducida por el uso novedoso de las TIC, y se evidencia una mejora del aprendizaje adquirido durante la experiencia vivida en comparación al presentado antes de esta. 
Similares resultados obtiene Salazar (2019) cuando afirma que esta metodología mejora el trabajo colaborativo, la participación y la comunicación entre los actores implicados.

Durante la implementación de nuevos métodos de enseñanza influyen diversos factores en el desarrollo cognitivo de los estudiantes. Las bajas no muy significativas que presentan los discentes del grupo experimental radican en la desmotivación hacia la lectura y el temor a desempeñar un rol como ente activo, responsable de crear sus conocimientos de manera autónoma. Tal como se evidencia en el indicador nivel de comprensión del lenguaje científico, para el cual los discentes presentan un nivel bajo en comparación al obtenido por el grupo de control. Estos hallazgos, junto con los de Broman y Johnels (2019), hacen que este factor de temor a ejercer un rol activo aparezca como uno de los principales obstáculos a tener en cuenta a la hora de implementar la metodología de aula invertida en la enseñanza-aprendizaje de la química.

\section{Referencias}

Bastidas, T. B. (2020). Aplicación del modelo pedagógico: Aula invertida y su incidencia en el aprendizaje significativo de quimica en los estudiantes del primer año de B.G.U. de la U.E.M. "Sebastián de Benalcázar" de la ciudad de Quito, periodo 2018-2019 [Tesis de maestría. Universidad Tecnológica Iberoamericana, Quito]. https://r.issu.edu.do/l?1=6744LY

Bergmann, J., \& Sams, A. (2012). Flip your Classroom: Reach Every Student in Every Class Every Day. Colorado: ISTE.

Broman, K., \& Johnels, D. (2019). FFlipping the Class - University Chemistry Students' Experiences from a new Teaching and Learning Approach. Chemistry Teacher International, 1(1), 1-8. https://doi.org/10.1515/cti-2018-0004

Chaljub Hasbún, J. (2017). El b-Learning y la clase para el desarrollo del aprendizaje activo, la autogestión y el pensamiento crítico universitario. Cuaderno de Pedagogía Universitaria, 13(26), 36-47. https://doi.org/10.29197/cpu.v13i26.262
Cruz C., L. M. (2018). Impacto del uso de las TICs en la educación superior dominicana. Revista Rido. https://r.issu.edu.do/l?1=687QP5

Eichler, J. F., \& Peeples, J. (2019). Flipped Classroom Learning Environments in General Chemistry: What is the Impact on Student Performance in Organic Chemistry? ACS Symposium Series, 1322, 171-189.

https://doi.org/10.1021/bk-2019-1322.ch010

Eldy, E. (2019). Inverted classroom improves pre-university students understanding on basic topic of Physics: The preliminary study. Journal of Technology and Science Education, 9(3), 420-427. https://doi.org/10.3926/jotse.599

Flavell, J. H. (1985). El desarrollo cognitivo. Universidad de Stanford: Prentice-Hall.

Joksimović, D., Veg, A., Simonović, D., Regodić, M., Šiniković, B., \& Gubeljak, N. (2019). Implementation of inverted classroom methodology in 3D modeling course. FME Transactions, 47, 310-315.

Ministerio de Educación (2016). Diseño Curricular Nivel Secundario, Segundo Ciclo. Santo Domingo. https://r.issu.edu.do/l? $=677 \mathrm{vKb}$

Ministerio de Educación (2017). Informe curricular de pruebas nacionales, Ciencias de la Naturaleza. Informe curricular de los resultados de las Pruebas Nacionales, primera y segunda convocatorias. Departamento de Pruebas Nacionales, Santo Domingo. https://r.issu.edu.do/l?1=6766rc

Ministerio de la Presidencia (2020). República Digital. Santo Domingo. https://bit.ly/2KxPtdi

Muñoz, S. A. (2014). Aula tradicional vs aula invertida. España: Procomún. https://r.issu.edu. $\mathrm{do} / \mathrm{l} ? \mathrm{l}=709 \mathrm{~h} 2 \mathrm{~L}$

Piaget, J. (1970). La teoría de Piaget: Monografías de infancia y aprendizaje (pp. 703-732). Nueva York: John Wiley and Sons.

Quiroga, A. (2014). Observatorio de Educación: Definición de aula invertida. Politécnico Gran Colombiano. 


\section{JATO-CANALES • FAUSTO-FRÍAS • DOMÍNGUEZ-LIRIANO}

Aula invertida como método de enseñanza en la unidad didáctica Reacciones Químicas de quinto...

Salazar, J. C. (2019). Aula invertida como metodología educativa para el aprendizaje de la Quimica en Educación Media [tesis de maestría. Universidad de la Costa CUC]. Colombia. https://r.issu.edu.do/l?1=688cF6

Sánchez-Cruzado, C. (2017). Flipped Classroom. La clase invertida, una realidad en la Facultad de Ciencias de la Educación de la Universidad de Málaga [Tesis doctoral. Universidad de Málaga]. Málaga. https://r.issu.edu.do/l?l=68129h

Sierra, E. J., Dimas, J. M., \& Flórez, E. P. (2018). Evaluación del uso del método Flipped Classroom o aula invertida en el aprendizaje de la química: Estudio de caso en la Institución
Educativa Lacides C. Bersal de Lorica. Revista Tecné, Episteme y Didaxis (número extraordinario), 1-9. https://r.issu.edu.do/l?l=68261P

UNESCO (2016). Innovación educativa. Lima: Unesco. https://r.issu.edu.do/l?!=710Uvd

Woolfolk, A. (2010). Psicología educativa. México: Pearson Educación de México.

Yarleque, J. V. (2018). Flipped classroom y el efecto en las competencias transversales de los alumnos del curso de electricidad electrónica [Tesis de grado. Universidad Peruana Cayetano Heredia]. Perú. https://r.issu.edu.do/l?1=684v7o

\section{CÓMO CITAR:}

Jato-Canales, S., Fausto-Frías, S., \& Domínguez-Liriano, J. D. (2021). Aula invertida como método de enseñanza en la unidad didáctica Reacciones Químicas de quinto grado del Nivel Secundario. RECIE. Revista Caribeña de Investigación Educativa, 5(1), 19-39. https://doi.org/10.32541/recie.2021.v5i1.pp19-39 